- This document may contain data, which exceeds the sheet parameters. It was furnished in this condition by the organizational source and is the best copy available.

- This document may contain tone-on-tone or color graphs, charts and/or pictures, which have been reproduced in black and white.

- This document is paginated as submitted by the original source.

- Portions of this document are not fully legible due to the historical nature of some of the material. However, it is the best reproduction available from the original submission.

Produced by the NASA Center for Aerospace Information (CASI) 


\section{THE EFFECTS OF NUCLEAR FORCES ON THIE MAXIMUM MASS LIMIT OF NEUTRON STARS}

\section{SACHIKO TSURUTA}

SEPTEMBER 1970

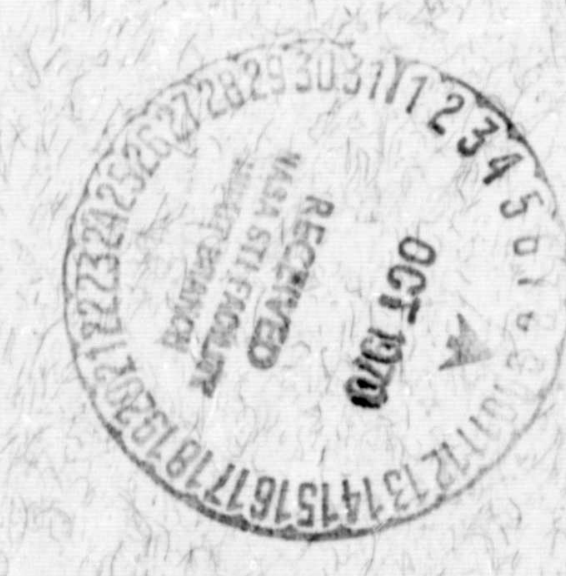

GSFC - GODDARD SPACE FLIGHT CENTER

GREENBELT, MARYLAND
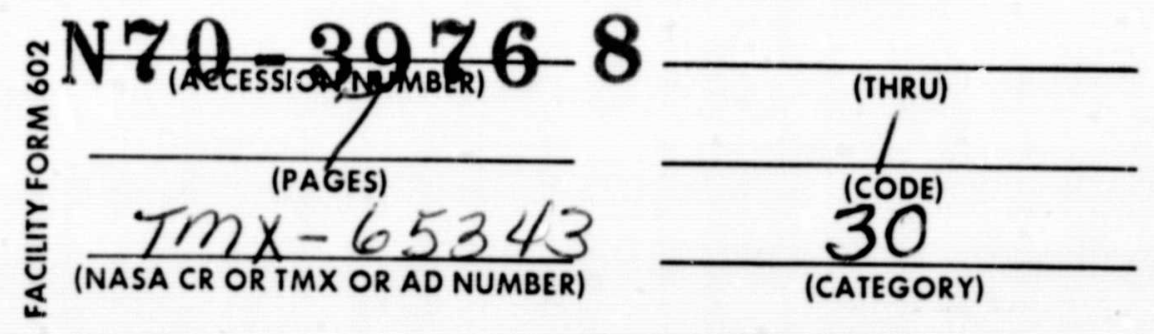
THE EFFECTS OF INUCLEAR FORCES ON THE MAXIMUM MASS LIMIT

OF NEUTRON STARS*

by

SACHIKO TSURUSA**

Goddard Space Fli ght Center

Greenbelt, Maryland

Recently the problem of the maximum rass limit of a stable neutron star has drawn the attention of many people, because it may seriously affect the models of pulsars and other phenomena which are most likely caused by the presence of neutron stars. In this brief report I wish to cumpare the neutron star models by various groups, including the most recent results I am aware of, those by the Cornell group (Boozer-Salpeter) and those by the Kyoto group (S. Ikeuchi, T. Mizutani, S. Nagata, R. Tamagaki, nd C. Hayashi.).

In Figure 1, the curve marked IDEAL represents the models with no nuclear interactions, originally constructed by Oppenheimer and Volkoff. ${ }^{1}$ The models by Harrison, Thorne, Wakano and Wheeler ${ }^{2}$ approxin.tely lie on the same curve in the neutron star region. The dotted curves marked $v_{\beta}$ and $V_{Y}$ are the models constructed by Tsuruta and Cameron ${ }^{3}$ and subsequently used by Thorne and others at $\mathrm{CIT}^{4}$ in their calculations of the moments of inertia of neutron stars, etc. I decided to show these curves also, because these results (especially the $V_{\gamma}$ models) have been used by Ostriker and others in their pulsar studies. The solid curves show neutron star models

* Paper presented at the IAU Symposium No. 46 "The Crab Nebula," Nuffield Radio Astranomy Laboratories, Jodrell Bank, England, August 5-7, 1970.

** NAS-NRC Research Associate presęntly at Goddard Space Flight Center. 
constructed after the pulsar discovery. The curve (1) is by Cohen et al. ${ }^{6}$, (2) is by the Cornell group (Boozer-Salpeter), (3) is by Wang et al. ${ }^{7}$, and the curves (4) through (7) are obtained by the Kyoto group.

In all models shown here except the first (those marked IDEAL), nuclear interactions are taken into account. In the models marked as $V_{\beta}$ and $V_{\gamma}$, the nuclear potentials of $\mathrm{V}_{\beta}$ and $\mathrm{V}_{\gamma}$ type, respectively, by Levinger and Simmons $^{8}$ are used. In the models (1), the moaified Levinger-Simmons neutron gas models are used. Boozer and Salpeter (the curve (2)) made use of the neutron gas calculations (2b) by Nemeth and Sprung ${ }^{9}$ with the soft-core Reid potentials. Wang et al. (the curve (3)) took the average of the soft-core Reid potentials anả several other potentials, but they all give the similar equations of state. The results by the Kyoto group are obtained in the following way. For densities $\rho$ less than $\rho_{0} \simeq 8 \times 10^{i 4} \mathrm{gm} / \mathrm{cm}^{3}$ where the neutron matter calculations from the concept of "nuclear potential" becomes unreliable, the one-boson exchange hard-core potentials constructed by Kishi were used in the models (4), called OBEP-K, and the one-pion exchange potentials with a Gaussian type soft core constructed by Tamagaki were used in the models (5) called OPEG-T. For $\rho>\rho_{0}$ in these models, the equation of state obtained in this manner was paraholically extrapolated in the logarithmic scale. In the models (6) and (7), the equation of state $P=\epsilon / 3$ and $P=\epsilon$, respectively, (where $\epsilon$ is the energy density) were used for $\rho>\rho_{1} \approx 5 \times 10^{15} \mathrm{~cm} / \mathrm{cm}^{3}$ where the Zel'dovich type scalar or vector interactions are assumed to become applicable, the method (4) was used for $\rho \lesssim \rho_{0}$, and the intermediate regions have been interpolated. Similar results are obtained if the OPEG-T or the Hamada-Johnston potentials are used for $\rho \lesssim \rho_{0}$. 
We see, first of all, that drastically different masses are obtained depending on how the inter-particle interaction problem is treated. In the models (2) and (3) the calculations are terminated where the nuclear potential approach is thought to become unreliable. If the calculations are extended to higher densities, the curve (2) reaches the mass peak of aioout $1 \mathrm{M} \odot$, similar to the curves (4) and (5) by the Kyoto group. Near the breakdown point, $\rho_{0}$, the models $(3),(4),(5),(6)$ and $V_{\beta}$ have small mass values of only around $0.2-0.4 \mathrm{M} \odot$, while the other models shown in the figure have larger masses. At the mass peak, the curve (7) reaches almost $3 M \odot$ - It may be pointed out that these models obtained by different methods nevertheless possess a few common points as mentioned below. As the mass increases from the minimum value to approximately $0.2 \mathrm{M} \odot$, the corresponding radius decreases quickly from about $300 \mathrm{~km}$ to about $10 \mathrm{~km}$ or so. This occurs a little below or around the nuclear density, depending on the models. For densities of around $10^{15} \mathrm{gm} / \mathrm{cm}^{3}$ the radius is around $10 \mathrm{~km}$, and for densities of around $10^{16} \mathrm{gm} / \mathrm{cm}^{3}$ the radius is around $5 \mathrm{~km}$. Our studies also show that the effects of the presence of hyperons and protons on the mass limit are much smaller than the effects of nuclear forces, if the interactions among all baryons are basically similar. It will be hard to draw definite conclusions from the above results, but the following comments may be valid.

In the regions where the concept of "nuclear potential" is still valid - (for approximately $\rho \lesssim 10^{15} \mathrm{gm} / \mathrm{cm}^{3}$ ), using realistic potentials alone is not enough. For instance, quite different results are obtained in the models (2) and (3), even though the both groups used the similar potentials (the soft-core Reid potentials). In this respect (of applying realistic potentials in a realistic way), I feel that the models (5), the OPEG-T, are the best recommended (among the models shown here). However, it may 
be noted that many-body interactions are generally neglected and that the nuclear potential term is treated non-relativistically in the work done so far. The net effect of the corrections to these approximations seems to lower the densities and increase the masses near the mass peak. Thus the $\mathrm{V}_{\gamma}$ models used already in various pulsar studies seem to be not far from reality.

The exact behavior in the regions above about $10^{15} \mathrm{gm} / \mathrm{cm}^{3}$ seems to be beyonc. the knowledge of present-day physics. Since the maximum mass of some of the models lie in these high density regions, i.t is hard to answer the question of how high masses stable neutron stars can have. It will be fortunate if such a question can be answered rather from the observational side. Will it be an impossible dream, if one contemplates that more detailed pulsar observations might help the break-through of the difficulties facing us today in particle physics and some other fields?

In the pre-pulsar discovery periods, I did not see much sense in going beyond our $\mathrm{v}_{\beta}$ and $\mathrm{v}_{\gamma}$ models of neutron stars. But, todar, with more observations of pulsars becoming available, better theoretical work with the cooperation of experts in various fields, including the effort to construct mort realistic neutron star models, seems very desirable.

I wish to thank Professors Hayashi, Nagata, Salpeter and Tamagaki, and Messrs. Boozer, Ikeuchi and Mizutani, for giving me the results of their calcuiations ${ }^{10}$ before publications. 


\section{REFERENCES}

1. Oppenhes.mer, J. R. and Volkoff, G. M., Phys. Rev., 25, 374, 1939.

2. Harrison, B. K., Thorne, K. S., Wakano, M. and Wheeler, J. A., Gravication Theory and Gravitational Collapse (University of Chicagn Press), 1965.

3. Tsuruta, S. and Cameron, A. G. W., Can. J. Phys., 44, 1895, 1966.

4. See, for instance, Hartle, J. B., and Thorne, K. S., Astrophys. J., 153, $807,1968$.

5. See, for instance, Ostriker, J. P. and Gunn, J. E., Astrophys. J., :j7, $1395,1969$.

6. Cohen, J. M., Langer, W. D., Rosen, L. C. and Cameron, A. G. W., Astrophys. Space Sci., $\underline{6}, 228,1970$.

7. Wang, C. G., Rose, W. K., and Schlenker, S. L., Astrophys. J. Lett., 160, L17, 1970.

8. Levinger, J. S. and Simmons, L. M., Phys. Rev., 124, 916, 1961.

9. Nemeth, J. and Sprung, D. W. L., Phys. Rev., 176, 1496, 1968.

10. To be published. Also Mizutani, T., M. A. Thesis, Tokyo University, unpublished, 1970. 


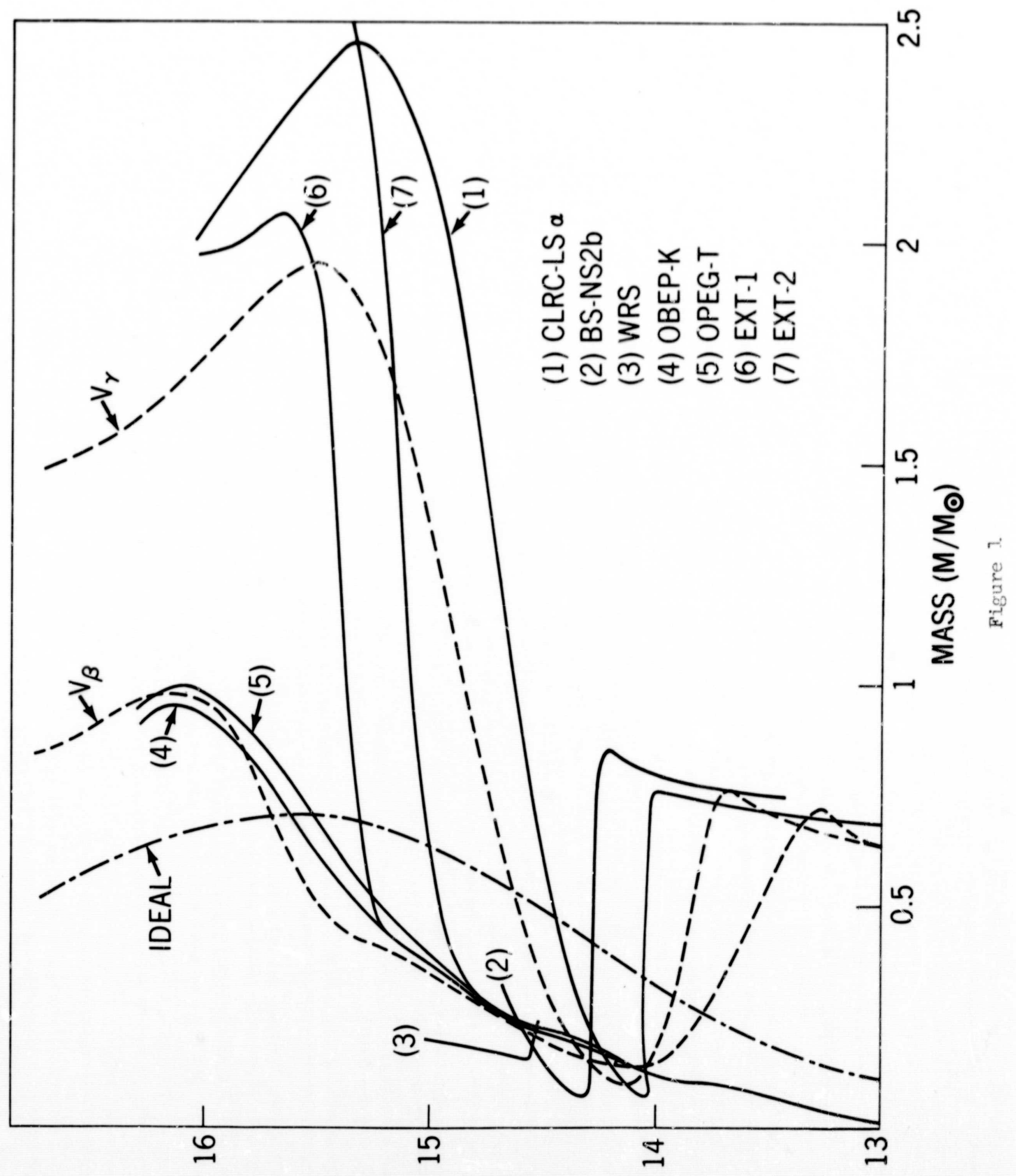

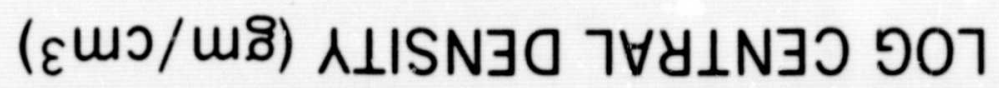

